\title{
Delayed onset of the diurnal melatonin rise in patients with Huntington's disease
}

\author{
N. Ahmad Aziz · Hanno Pijl · Marijke Frölich · \\ Janny P. Schröder-van der Elst · Chris van der Bent • \\ Ferdinand Roelfsema $\cdot$ Raymund A. C. Roos
}

Received: 22 April 2009/Accepted: 24 May 2009/Published online: 27 June 2009

(C) The Author(s) 2009. This article is published with open access at Springerlink.com

\begin{abstract}
Sleep disturbances are very prevalent in Huntington's disease (HD) patients and can substantially impair their quality of life. Accumulating evidence suggests considerable dysfunction of the hypothalamic suprachiasmatic nucleus $(\mathrm{SCN})$, the biological clock, in both HD patients and transgenic mouse models of the disease. As melatonin has a major role in the regulation of sleep and other cyclical bodily activities and its synthesis is directly regulated by the SCN, we postulated that disturbed SCN function is likely to give rise to abnormal melatonin secretion in HD. Therefore, we compared $24 \mathrm{~h}$ melatonin secretion profiles between early stage HD patients and age-, sex- and body mass index-matched controls. Although mean diurnal melatonin levels were not different between the two groups $(p=0.691)$, the timing of the evening rise in melatonin levels was significantly delayed by more than 01:30 h in HD patients $(p=0.048)$. Moreover, diurnal melatonin levels strongly correlated with both motor $(r=-0.70, \quad p=0.036)$ and functional impairment $(r=+0.78, p=0.013)$. These findings suggest a delayed sleep phase syndrome-like circadian rhythm disorder in
\end{abstract}

\section{N. A. Aziz $(\varangle)$ · R. A. C. Roos}

Department of Neurology, K-05-Q 110,

Leiden University Medical Center, P.O. Box 9600,

Albinusdreef 2, 2300 RC Leiden, The Netherlands

e-mail: n.a.aziz@lumc.nl

H. Pijl · J. P. Schröder-van der Elst · C. van der Bent .

F. Roelfsema

Department of Endocrinology and Metabolic Diseases,

Leiden University Medical Center, Leiden, The Netherlands

\section{Frölich}

Department of Clinical Chemistry,

Leiden University Medical Center,

Leiden, The Netherlands early stage HD patients and suggest that melatonin levels may progressively decline with advancing disease.

Keywords Huntington's disease - Melatonin - Sleep · Circadian rhythm $\cdot$ Hypothalamus

\section{Introduction}

Huntington's disease (HD) is an autosomal dominant neurodegenerative disorder caused by an expanded CAG repeat size in the gene encoding the protein huntingtin [4]. The disease is characterized by motor impairment, cognitive deterioration, behavioral problems and progressive weight loss [4]. With an estimated prevalence of nearly $90 \%$, disturbed sleep is also a prominent feature of the disease, substantially impairing the quality of life of both patients and caregivers [20]. Sleep disturbances in HD patients include an increased sleep onset latency, frequent nocturnal awakenings, reduced sleep efficiency, delayed and shortened rapid eye movement sleep, and increased periodic leg movements $[1,8,22,24]$. Moreover, wrist actigraphy studies indicate circadian rhythm disturbances in HD patients, which are mirrored in the most widely used transgenic mouse model of the disease, the R6/2 mouse $[10,14,15]$. The $\mathrm{R} 6 / 2$ mice show progressive disruption of the day-night activity patterns, with increased daytime activity and a concurrent decrease in nocturnal activity, eventually leading to a complete disintegration of the circadian behavior $[14,15]$. Interestingly, disrupted circadian behavior in these mice is accompanied by marked dysregulation of expression of a number of circadian clock genes in the hypothalamic suprachiasmatic nucleus (SCN), the principal rhythm generating system in mammals [12, 14, 15]. Furthermore, we recently demonstrated an 
increased amplitude of the diurnal cortisol profile as well as an increased rate of early day cortisol production in HD patients, both of which are also consistent with SCN dysfunction in HD [3].

Melatonin is a hormone that is primarily secreted at night by the pineal gland and has a major role in the regulation of sleep and other cyclical bodily activities $[5,16]$. Melatonin synthesis is directly regulated by the SCN via a multisynaptic pathway in response to the environmental light/dark cycle, and thus, melatonin is considered an endogenous humoral synchronizer that signals time of day' to all tissues throughout the body [5]. Conversely, the two major melatonin receptors, MT1 and MT2 receptors, are abundantly expressed in the SCN and are thought to mediate melatonin's sleep-promoting and circadian phaseshifting effects [5]. Therefore, we postulated that disturbed SCN function in HD is likely to give rise to abnormal melatonin secretion, which in turn could contribute to impaired sleep and circadian rhythm disturbances in HD patients. In addition, as apart from its timekeeping functions, melatonin also has strong antioxidative propertiesabnormal melatonin secretion may also influence the neurodegenerative process underlying HD. In order to test these hypotheses, we: (1) compared $24 \mathrm{~h}$ plasma melatonin concentration profiles between early stage, medication-free HD patients and healthy matched controls, and (2) assessed the association between mean diurnal melatonin levels and clinical phenotype.

\section{Subjects and methods}

\section{Subjects}

Nine early stage HD patients and nine healthy control subjects, matched for age, sex, and body mass index (BMI), were enrolled in the study. Clinical details are summarized in Table 1. The clinical diagnosis of HD was made by a neurologist specialized in movement disorders (R.A.C.R.). The unified Huntington's disease rating scale (UHDRS) was used to assess HD symptoms and signs [9]. All subjects were free of medication, except one HD patient who discontinued paroxetine use 3 weeks prior to the study. Written informed consent was obtained from all subjects. The study was approved by the ethics committee of the Leiden University Medical Center.

\section{Clinical protocol}

Subjects were admitted to the Clinical Research Center for $24 \mathrm{~h}$ blood sampling. Two women (one patient and one control) were postmenopausal, the other women were studied in the early follicular phase of their menstrual cycles.
Table 1 Characteristics of the study population

\begin{tabular}{llll}
\hline & HD patients $^{\mathrm{a}}$ & Controls $^{\mathrm{a}}$ & $p$-value $^{\mathrm{b}}$ \\
\hline Male/female & $6 / 3$ & $6 / 3$ & - \\
Age (year) & $47.1(3.4)$ & $48.6(3.3)$ & 0.691 \\
BMI & $24.1(1.0)$ & $24.3(0.6)$ & 0.691 \\
Mutant CAG repeat size & $44.4(1.0)$ & - & - \\
Disease duration (year) & $5.7(1.1)$ & - & - \\
UHDRS motor score & $22.2(6.0)$ & - & - \\
TFC score & $11.7(0.7)$ & - & - \\
Functional assessment & $23.3(0.7)$ & - & - \\
Independence score & $94.4(2.8)$ & - & - \\
\hline
\end{tabular}

BMI body mass index; FAS functional assessment; TFC total functional capacity; UHDRS unified Huntington's disease rating scale

${ }^{a}$ Values are indicated as mean (SE)

b Differences between groups were assessed by the Mann-Whitney $U$-test

A cannula was inserted into an antecubital vein $45 \mathrm{~min}$ before the start of blood sampling at 16:30 h. Blood samples were collected with S-monovetten (Sarstedt, Etten-Leur, The Netherlands) from a three-way stopcock that was attached to a $0.9 \% \mathrm{NaCl}$ and heparin $\left(1 \mathrm{U} \mathrm{ml}^{-1}\right)$ infusion $(500 \mathrm{ml} / 24 \mathrm{~h}$ ) to keep the cannula from clotting. Sampling was performed through a long line to prevent sleep disruption by investigative manipulations. During $24 \mathrm{~h}$, blood was collected in serum tubes every hour. Blood was allowed to clot and, within 60-min of sampling, centrifuged at 4,000 rotations $\min ^{-1}$ at $4^{\circ} \mathrm{C}$ for $20 \mathrm{~min}$, and plasma was stored at $-80^{\circ} \mathrm{C}$ until assay. Three standardized meals were served at 09:00, 13:00, and 19:00 h (Nutridrink, $1.5 \mathrm{kcal} \mathrm{ml}^{-1}, 1,500-1,800 \mathrm{kcal} \mathrm{d}^{-1}$; macronutrient composition per $100 \mathrm{ml}$ : protein, $5 \mathrm{~g}$; fat, $6.5 \mathrm{~g}$; carbohydrate, $17.9 \mathrm{~g}$; Nutricia, Zoetermeer, The Netherlands). Twentyfour hour urine was collected for the determination of creatinine and catecholamine concentrations. Subjects remained sedentary except for bathroom visits. No daytime naps were allowed. Lights were switched off at 23:00 h and back on at 07:30 the next morning.

\section{Assays}

Plasma melatonin was measured by radioimmunoassay (Labor Diagnostika Nord GmbH \& Co. KG, Nordhorn, GER). The detection limit of the assay was $2 \mathrm{pg} \mathrm{mL}^{-1}$. The intra-assay and interassay variations ranged from 9.8 to $12.3 \%$ and from 9.6 to $16.2 \%$, respectively. Samples from each patient and matched control were handled in the same run. Urine creatinine was measured by a fully automated $\mathrm{P}$ 800 Modular system (Roche, Almere, The Netherlands). Urinary epinephrine, norepinephrine and dopamine concentrations were assessed by high performance liquid 
chromatography with electron capture detection (ESTACoulochem, Chelmsford, MA, USA).

Analysis of melatonin profiles

Individual diurnal variations of plasma melatonin levels were quantified by a best-fit curve obtained using locally weighted linear regression with a Gaussian kernel and a regression window of $4 \mathrm{~h}$ as previously described [11]. For each melatonin profile the nadir and the acrophase were defined as the minimum and maximum of the best-fitting curve, while the amplitude was defined as half of the difference between the acrophase and nadir values. The onset of the melatonin rise was defined as the timing of the first plasma level exceeding the mean +3 standard deviations (SDs) of baseline levels recorded over the 1030-1430 period, not followed to lower concentrations before the acrophase. The melatonin offset was defined as the timing of the last value occurring after the acrophase that exceeded +3 SDs of the baseline values [11].

\section{Statistical analysis}

Results are expressed as mean \pm standard error (SE) unless otherwise specified. The non-parametric MannWhitney $U$-test was used to assess group differences. Spearman's correlation coefficient was applied to assess all correlations. All tests were two-tailed and significance level was set at $p<0.05$. Statistical analyses were performed using SPSS for Windows (release 16.0, SPSS, Inc., Chicago, IL).

\section{Results}

\section{Subjects}

The HD and the control group did not differ with respect to age, sex, and BMI (all $p \geq 0.691$, Table 1). There were also no significant differences in urinary creatinine, epinephrine, norepinephrine and dopamine levels (all $p \geq 0.10$ ).

\section{Melatonin profiles}

Mean $24 \mathrm{~h}$ melatonin levels were not significantly different between HD patients and controls $(24.8 \pm 5.4$ vs. $22.7 \pm 2.8 \mathrm{pg} \mathrm{mL}^{-1}, p=0.691$; Fig. 1). Also the acrophase and nadir concentrations, as well as the amplitude of the diurnal melatonin profile were not significantly different between the two groups ( $p \geq 0.857$; Table 2$)$. In one HD patient, however, the diurnal melatonin profile was extremely irregular and at no point did melatonin concentrations

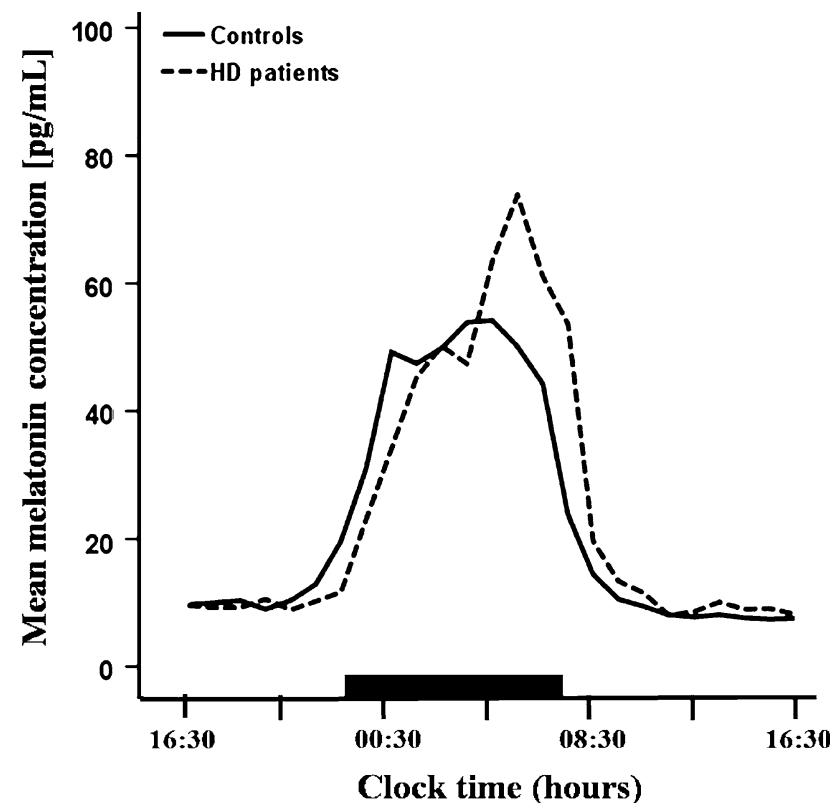

Fig. 1 Mean $24 \mathrm{~h}$ melatonin levels in HD patients and matched controls. The diurnal melatonin rise was significantly delayed in HD patients by about $01: 30 \mathrm{~h}(p=0.048)$. The black bar on the abscissa indicates the dark period $(23: 00-7: 30 \mathrm{~h})$

rise above three standard deviations of the mean baseline values (this subject also happened to be the most severely affected patient with scores of 63 and 7 on the UHDRS motor and total functional capacity subscales, respectively). Consequently, melatonin onset, offset and duration could not be defined in this subject; therefore, for subsequent comparison of these parameter values between the two groups, the data of the subject and the matched control subject were excluded from the analyses. Melatonin onset time was significantly delayed in HD patients compared with controls $(00: 30 \mathrm{~h} \pm$ 22 min vs. $22: 52 \mathrm{~h} \pm 37 \mathrm{~min}, p=0.048$ ). Melatonin offset time, however, was similar between the two groups

Table 2 Melatonin secretion characteristics in HD patients and controls

\begin{tabular}{|c|c|c|c|}
\hline & HD patients ${ }^{\mathrm{a}}$ & Controls $^{\mathrm{a}}$ & $\begin{array}{l}p- \\
\text { value }^{b}\end{array}$ \\
\hline Mean $24 \mathrm{~h}$ levels $\left(\mathrm{pg} \mathrm{mL}^{-1}\right)$ & $24.8(5.4)$ & $22.7(2.8)$ & 0.601 \\
\hline Acrophase conc. $\left(\mathrm{pg} \mathrm{mL}^{-1}\right)$ & $76.6(20.4)$ & $60.1(9.7)$ & 0.860 \\
\hline Nadir conc. $\left(p g \mathrm{~mL}^{-1}\right)$ & $5.8(0.9)$ & $5.8(0.7)$ & 0.857 \\
\hline Amplitude (pg mL $\left.{ }^{-1}\right)$ & $35.4(9.9)$ & $27.2(4.8)$ & 0.895 \\
\hline Onset time (hh:mm) & $00: 30(00: 22)$ & $22: 52(00: 40)$ & $0.048^{*}$ \\
\hline Offset time (hh:mm) & 07:30 (00:48) & $08: 22(00: 21)$ & 0.478 \\
\hline Nocturnal duration (h) & $7.0(1.0)$ & $9.5(0.8)$ & 0.063 \\
\hline \multicolumn{4}{|l|}{$p<0.05$} \\
\hline${ }^{\text {a }}$ Values are indicated as me & $\operatorname{an}(\mathrm{SE})$ & & \\
\hline
\end{tabular}


(Table 2). Consequently, there was also a trend for a shorter duration of the nocturnal plasma melatonin plateau in HD patients $(6.2 \pm 1.2$ vs. $9.1 \pm 0.8 \mathrm{~h}, p=0.063)$.

Melatonin levels and clinical phenotype

In $\mathrm{HD}$ patients, mean $24 \mathrm{~h}$ melatonin levels significantly correlated with UHDRS motor score $(r=-0.70, p=$ $0.036)$, total functional capacity $(r=+0.78, p=0.013)$, and independence score $(r=+0.88, p=0.002)$, but not with mutant CAG repeat size $(r=+0.18, p=0.645)$.

\section{Discussion}

Here we present the first detailed description of diurnal melatonin profiles in HD patients. We show that the timing of the evening rise in melatonin levels is significantly delayed by more than 01:30 $\mathrm{h}$ in these patients compared with matched control subjects. Moreover, despite similar mean diurnal melatonin levels between our early stage HD patients and controls, we found strong inverse associations between mean diurnal melatonin levels and both motor and functional disability in these patients, suggesting that decreases in melatonin levels are likely to become more pronounced in the later stages of the disease.

Delayed onset of melatonin secretion in HD patients is reminiscent of a delayed sleep phase syndrome (DSPS)like circadian rhythm disorder [25]. The pathophysiological basis of DSPS is assumed to lie in a slower endogenous clock with an abnormally long intrinsic circadian periodicity, resulting in a delayed phase position of the overt circadian rhythms, including those of melatonin, cortisol and core body temperature [25]. Interestingly, recently we also found an increased rate of early day cortisol production in HD patients, which may also be a manifestation of delayed circadian rhythms in HD [3]. Circadian rhythm disturbances in HD are likely to stem directly from pathology within the SCN molecular oscillation, caused either by the toxic effects of mutant huntingtin locally and/ or arising from dysfunction of brain circuitry afferent to the $\mathrm{SCN}[14,15]$. In favour of local pathology is the detection of neuronal inclusions of mutant huntingtin in the SCN of HD patients [2], as well as the finding of SCN dysfunction at both mRNA and protein level in $\mathrm{R} 6 / 2$ mice, with reduced levels of the positive regulator mBmall and truncated peak expression of its target genes mPerl, mPer2, and mProk2 [14]. However, intact oscillation of SCN neurons from R6/2 mice in vitro, when released from the pathological context, is consistent with an afferent cause of SCN dysfunction in HD [15]. Alternatively, impaired expression and/or function of the melatonin receptors in HD could play a role, particularly the MT2 receptor subtype which is enriched in the $\mathrm{SCN}$ and is known to be involved in phase-shifting of the biological clock [17]. Regardless of the cause of diurnal rhythm disturbances in HD, however, restoration of circadian rhythms by pharmacological imposition of sleep has been shown to improve cognitive decline in R6/2 mice, suggesting that a similar strategy may be beneficial to HD patients. As our findings indicate a DSPS-like phenotype in early stage HD, another approach that may be evaluated in these patients is to treat them with melatonin and/or bright light at the appropriate times so as to phase advance the clock [13, 25]. The administration of melatonin at the subjective dusk, and the use of bright light at the subjective dawn and avoidance of light in the subjective evening, could be used to phase advance the clock [13, 25].

Interestingly, we also found that mean diurnal melatonin levels in HD patients decreased with increasing severity of the clinical phenotype, suggesting that melatonin levels may decline substantially with advancing disease course. Progressive abnormalities in the metabolism of the melatonin precursor tryptophan may account for this association [7, 19], although additional investigations are needed to pinpoint the exact underlying metabolic pathways. Numerous studies have shown the ability of melatonin and its kynuramine metabolites to increase the survival of neurons under conditions of enhanced oxidative stress [18]. Therefore, declining melatonin levels may contribute to the progressive neurodegeneration in HD, and conversely, exogenous melatonin supplementation may be of benefit to HD patients. In fact, melatonin can antagonise the cytotoxic properties of both quinolinic acid and 3-nitropropionic acid, the administration of which is used to model HD induced pathology [6, 21]. Moreover, recently it was demonstrated that melatonin can potently inhibit mitochondrial cytochrome $c$ release, which is known to activate downstream cell death pathways, resulting in neuroprotection in a mutant huntingtin expressing striatal cell line [23].

In conclusion, our findings suggest a DSPS-like circadian rhythm disorder in early stage HD patients and indicate that melatonin levels may progressively decline with advancing disease. Therefore, strategies aimed at advancing the phase of the biological clock as well as melatonin supplementation might be of benefit to HD patients. First, however, larger scale studies are needed to confirm our findings and to assess whether later stages of HD are also accompanied by a similar circadian rhythm disorder.

Acknowledgments We are greatly indebted to: Prof. Dr. J. H. Meijer for her valuable suggestions and comments, and E. J. M. Ladan-Eygenraam, and E. C. Sierat-van der Steen for technical assistance during the study. N. A. Aziz is supported by The Netherlands Organisation for Scientific Research (grant \#017.003.098). 
Conflict of interest statement There are no conflicts of interest to be disclosed.

Open Access This article is distributed under the terms of the Creative Commons Attribution Noncommercial License which permits any noncommercial use, distribution, and reproduction in any medium, provided the original author(s) and source are credited.

\section{References}

1. Arnulf I, Nielsen J, Lohmann E, Schieffer J, Wild E, Jennum P et al (2008) Rapid eye movement sleep disturbances in Huntington disease. Arch Neurol 65(4):482-488. doi:10.1001/ archneur.65.4.482

2. Aziz A, Fronczek R, Maat-Schieman M, Unmehopa U, Roelandse F, Overeem S et al (2008) Hypocretin and melaninconcentrating hormone in patients with Huntington disease. Brain Pathol 18(4):474-483

3. Aziz NA, Pijl H, Frolich M, van der Graaf AW, Roelfsema F, Roos RA (2009) Increased hypothalamic-pituitary-adrenal axis activity in Huntington's disease. J Clin Endocrinol Metab 94(4):1223-1228

4. Bates G, Harper PS, Jones L (2002) Huntington's disease, 3rd edn. Oxford University Press, New York

5. Benarroch EE (2008) Suprachiasmatic nucleus and melatonin: reciprocal interactions and clinical correlations. Neurology 71(8):594-598. doi:10.1212/01.wnl.0000324283.57261.37

6. Cabrera J, Reiter RJ, Tan DX, Qi W, Sainz RM, Mayo JC et al (2000) Melatonin reduces oxidative neurotoxicity due to quinolinic acid: in vitro and in vivo findings. Neuropharmacology 39(3):507-514. doi:10.1016/S0028-3908(99)00128-8

7. Christofides J, Bridel M, Egerton M, Mackay GM, Forrest CM, Stoy N et al (2006) Blood 5-hydroxytryptamine, 5-hydroxyindoleacetic acid and melatonin levels in patients with either Huntington's disease or chronic brain injury. J Neurochem 97(4):1078-1088. doi:10.1111/j.1471-4159.2006.03807.x

8. Emser W, Brenner M, Stober T, Schimrigk K (1988) Changes in nocturnal sleep in Huntington's and Parkinson's disease. J Neurol 235(3):177-179. doi:10.1007/BF00314313

9. Huntington Study Group (1996) Unified Huntington's disease rating scale: reliability and consistency. Huntington study group. Mov Disord 11(2):136-142. doi:10.1002/mds.870110204

10. Hurelbrink CB, Lewis SJ, Barker RA (2005) The use of the Actiwatch-Neurologica system to objectively assess the involuntary movements and sleep-wake activity in patients with mildmoderate Huntington's disease. J Neurol 252(6):642-647. doi: 10.1007/s00415-005-0709-z

11. Leproult R, Van Onderbergen A, L'hermite-Baleriaux M, Van Cauter E, Copinschi G (2005) Phase-shifts of 24-h rhythms of hormonal release and body temperature following early evening administration of the melatonin agonist agomelatine in healthy older men. Clin Endocrinol (Oxf) 63(3):298-304. doi:10.1111/ j.1365-2265.2005.02341.x
12. Meijer JH, Rietveld WJ (1989) Neurophysiology of the suprachiasmatic circadian pacemaker in rodents. Physiol Rev 69(3):671-707

13. Morgenthaler TI, Lee-Chiong T, Alessi C, Friedman L, Aurora RN, Boehlecke B et al (2007) Practice parameters for the clinical evaluation and treatment of circadian rhythm sleep disorders. An American academy of sleep medicine report. Sleep 30(11):14451459

14. Morton AJ, Wood NI, Hastings MH, Hurelbrink C, Barker RA, Maywood ES (2005) Disintegration of the sleep-wake cycle and circadian timing in Huntington's disease. J Neurosci 25(1):157163. doi:10.1523/JNEUROSCI.3842-04.2005

15. Pallier PN, Maywood ES, Zheng Z, Chesham JE, Inyushkin AN, Dyball R et al (2007) Pharmacological imposition of sleep slows cognitive decline and reverses dysregulation of circadian gene expression in a transgenic mouse model of Huntington's disease. J Neurosci 27(29):7869-7878. doi:10.1523/JNEUROSCI.064907.2007

16. Pandi-Perumal SR, Srinivasan V, Maestroni GJ, Cardinali DP, Poeggeler B, Hardeland R (2006) Melatonin: nature's most versatile biological signal? FEBS J 273(13):2813-2838. doi: 10.1111/j.1742-4658.2006.05322.x

17. Pandi-Perumal SR, Trakht I, Srinivasan V, Spence DW, Maestroni GJ, Zisapel N et al (2008) Physiological effects of melatonin: role of melatonin receptors and signal transduction pathways. Prog Neurobiol 85(3):335-353. doi:10.1016/j. pneurobio.2008.04.001

18. Srinivasan V, Pandi-Perumal SR, Cardinali DP, Poeggeler B, Hardeland R (2006) Melatonin in Alzheimer's disease and other neurodegenerative disorders. Behav Brain Funct 2:15. doi: 10.1186/1744-9081-2-15

19. Stoy N, Mackay GM, Forrest CM, Christofides J, Egerton M, Stone TW et al (2005) Tryptophan metabolism and oxidative stress in patients with Huntington's disease. J Neurochem 93(3):611-623. doi:10.1111/j.1471-4159.2005.03070.x

20. Taylor N, Bramble D (1997) Sleep disturbance and Huntingdon's disease. Br J Psychiatry 171:393. doi:10.1192/bjp.171.4.393c

21. Tunez I, Montilla P, Del Carmen MM, Feijoo M, Salcedo M (2004) Protective effect of melatonin on 3-nitropropionic acidinduced oxidative stress in synaptosomes in an animal model of Huntington's disease. J Pineal Res 37(4):252-256. doi:10.1111/ j.1600-079X.2004.00163.X

22. Videnovic A, Leurgans S, Fan W, Jaglin J, Shannon KM (2009) Daytime somnolence and nocturnal sleep disturbances in Huntington disease. Parkinsonism Relat Disord 15:471-474. doi: 10.1016/j.parkreldis.2008.10.002

23. Wang X, Zhu S, Pei Z, Drozda M, Stavrovskaya IG, Del Signore SJ et al (2008) Inhibitors of cytochrome c release with therapeutic potential for Huntington's disease. J Neurosci 28(38):9473-9485. doi:10.1523/JNEUROSCI.1867-08.2008

24. Wiegand M, Moller AA, Lauer CJ, Stolz S, Schreiber W, Dose M et al (1991) Nocturnal sleep in Huntington's disease. J Neurol 238(4):203-208. doi:10.1007/BF00314781

25. Zisapel N (2001) Circadian rhythm sleep disorders: pathophysiology and potential approaches to management. CNS Drugs 15(4):311-328. doi:10.2165/00023210-200115040-00005 